\title{
Babesia microti
}

National Cancer Institute

\section{Source}

National Cancer Institute. Babesia microti. NCI Thesaurus. Code C122180.

A species of intraerythrocytic protozoan parasites in the family Babesiidae. B. microti is transmitted by the tick Ixodes scapularis and is the primary causative agent of human babesiosis. 